\title{
Gastroschisis: Predicting outcomes through prenatal imaging and the current state of care in a single institution
}

Fisher, Sarah $\mathrm{G}^{3}$; Anderson, Cassandra $\mathrm{M}^{1}$; Steinhardt, Nicole $\mathrm{P}^{2}$; Brown, Brandon P MD2; Gray, Brian W MD ${ }^{1}$

${ }^{1}$ Section of Pediatric Surgery, Riley Hospital for Children, Department of Surgery; ${ }^{2}$ Riley Hospital for Children, Department of Radiology; ${ }^{3}$ Indiana University School of Medicine

Background: Gastroschisis occurs in 1 out of 2,000 births with survival rates partially contingent on intestinal complications and time to establishing feeding. Enhancements in prenatal imaging have given better insight into postnatal outcomes. The goal of this study is to examine the gastroschisis patient population and changes to care at Riley Hospital for Children in the modern era from 2010 to 2018. Additionally, we aim to utilize prenatal imaging to develop new prenatal prognostic indicators.

Methods: We performed a retrospective review of gastroschisis patients at Riley Hospital for Children from 2010 through 2018. We recorded demographics, prenatal data and imaging, early postnatal data, operative data, and patient outcomes and compared these variables to historic data gathered from studies at the same institution from the 2003-2009. Prenatal ultrasound factors were evaluated as a tool to predict the status of the bowel at birth. Statistical analyses were performed with chi square, univariate, and multivariate analyses.

Results: 134 patients were included in the study: complex (21), non-complex (113). Compared to non-complex gastroschisis, complex patients required longer median days to feeding initiation (46 vs $10, p<0.001$ ), full feeding (77.5 vs $23, p<0.001$ ), length of stay (LOS) ( 83 vs 33, $p<0.001$ ), and TPN at discharge $(p=0.031)$. Prenatal ultrasound factors showed a significant relationship between increased bowel dilation and non-complex gastroschisis $(p=0.015)$. Compared to 2003-2009, 2010-2018 data showed a significant decrease in mortality $(p=0.002)$ and use of primary closure $(p=0.050)$. Multivariable logistic regression revealed complex gastroschisis to be predictive of total intubation days in the first 30 days of life, LOS, time to initial feeds, and time to full feeding $(p<0.001)$.

Conclusion and Potential Impact: An increase in bowel dilation on prenatal ultrasound can be used to predict non-complex gastroschisis at birth. Complex gastroschisis is associated with increased time to feeds, LOS, and intubation days. 